\title{
Les retraites de rédaction structurées auprès des doctorant[e]s : Quelles conditions favorisent une expérience de rédaction légitime, productive et plaisante?
}

Émilie Tremblay-Wragg

Université du Québec à Montréal

Cynthia Vincent

Université du Québec à Montréal

Christelle Lison

Universition de Sherbrooke

William Gilbert

Université Laval

Philippe Valois

Université du Québec à Montréal

Sara Mathieu-Chartier

Université de Montréal

Canadian Journal of Education / Revue canadienne de l'éducation 44:2 (2021)

(O2021 Canadian Society for the Study of Education/ Société canadienne pour l'étude de l'éducation 


\title{
Résumé
}

La rédaction de la thèse est problématique pour nombre de doctorant[e]s. Pour les soutenir, la retraite de rédaction structurée semble une stratégie intéressante, mais peu de recherches ont tenté de la décrire. Cet article présente des résultats issus d'une étude mixte $(N=210)$, combinant questionnaires et entretiens pour mesurer et comprendre les effets des retraites Thèsez-vous en vue 1) d'en recenser les bénéfices, soit favoriser la productivité, légitimer la rédaction et vivre une expérience plaisante, et 2) d'identifier les conditions qui favorisent ces bénéfices. Ultimement, cet article contribue à l'identification de stratégies pour repenser et améliorer l'encadrement de la rédaction de thèse.

Mots-clés : rédaction, retraite de rédaction, bénéfices, flow, productivité scientifique

\begin{abstract}
Thesis writing is challenging for many $\mathrm{PhD}$ candidates. To support them, structured writing retreats represent a strategy for consideration, although little has been documented on the topic. This article presents results from a mixed-method study $(N=210)$ combining questionnaires and interviews, aiming to understand the effects of Thesez-vous writing retreats to 1) document benefits of writing retreats, namely encouraging writing, legitimizing writing and ensuring a fulfilling experience, and to 2) identify conditions maximizing these benefits. More broadly, this article conveys the possibility to rethink and reframe thesis writing to create a productive, collective and pleasant experience.
\end{abstract}

Keywords: thesis editing, writing retreats, benefits, flow, scientific productivity 


\section{Contexte}

Le parcours doctoral est une démarche basée sur un processus qui demande de nombreuses heures de rédaction et qui aboutit à un produit final, la thèse. Si dans les débuts du parcours universitaire les doctorant[e]s côtoient régulièrement leurs pairs, l'isolement caractérise la suite de leur parcours, surtout en période de rédaction (Aitchison et Guerin, 2014 ; Levecque et al., 2017). Cette période difficile entraine souvent une prolongation, voire un abandon des études (Litalien et Guay, 2015).

D'une part, plus le temps s'étire, plus l'étudiant[e] risque de faire face à des difficultés. Notamment, l'étudiant[e] devra peut-être reprendre certaines étapes ou sections de la thèse, comme une recension d'écrits plus récents ou la rédaction de sections qui ne sont plus à jour. Il convient de se demander si une production plus rapide et soutenue du projet doctoral permettrait d'éviter certaines prolongations, voire des abandons. D'autre part, comme le soulève Larivière (2012), les étudiant[e]s gradué[e]s participent grandement à la production scientifique au Québec, générant environ le tiers des publications en tant que premier[-ière] auteur[e] ou coauteur[e], fraction d'autant plus élevée avec la multiplication des thèses par articles (Mason et al., 2020). Les doctorant[e]s constituent une importante source de production de savoirs dans différents domaines. Pourtant, bon nombre vivent de la difficulté dans le processus d'écriture (Zaki, 2006), que ce soit en termes de structuration des idées, de leur articulation ou de leur enchainement, au point parfois d'abandonner à cette étape. Or, l'abandon des études supérieures occasionne des couts humains et financiers, tant pour les étudiant[e]s que pour les universités et le gouvernement (Denis, 2020 ; Litalien et Guay, 2015). Ainsi, les effets négatifs de l'abandon et de la prolongation des études doctorales sur les plans individuel et collectif poussent à réfléchir aux stratégies à mettre en place pour soutenir les étudiant[e]s durant leur processus de rédaction.

C'est en réponse à cette problématique que l'organisme à but non lucratif (OBNL) Thèsez-vous est né en 2015, avec la mission de soutenir la rédaction et de prévenir la prolongation et l'abandon aux cycles supérieurs en proposant des environnements sociaux et physiques favorables au bienêtre et à la productivité avec une approche par les pairs (Tremblay-Wragg et al., 2020). Parmi les services offerts, Thèsez-vous organise des retraites de rédaction de trois jours dont la structure est résumée à la figure 1. Plusieurs méthodes pédagogiques ont été adaptées et imbriquées dans la structure de la retraite de 
rédaction. Les principales sont 1) la fixation d'objectifs de rédaction S.M.A.R.T. en amont de la retraite, qui implique un découpage de la tâche en objectifs Spécifiques, Mesurables, Atteignables, Réalistes et Temporellement définis ; 2) l'affichage et le monitorage des objectifs individuels sur un babillard collectif suivant la méthode Kanban qui permet de visualiser sa progression ; 3 ) la gestion du temps selon une alternance de périodes de rédaction focalisée (50 minutes) et de pause (10 minutes) inspirée de la technique Pomodoro (pour plus de détails sur ces méthodes, voir Mathieu-Chartier et al., 2021).

\section{Figure 1}

Structure pédagogique des retraites de rédaction structurées organisées par Thèsez-vous

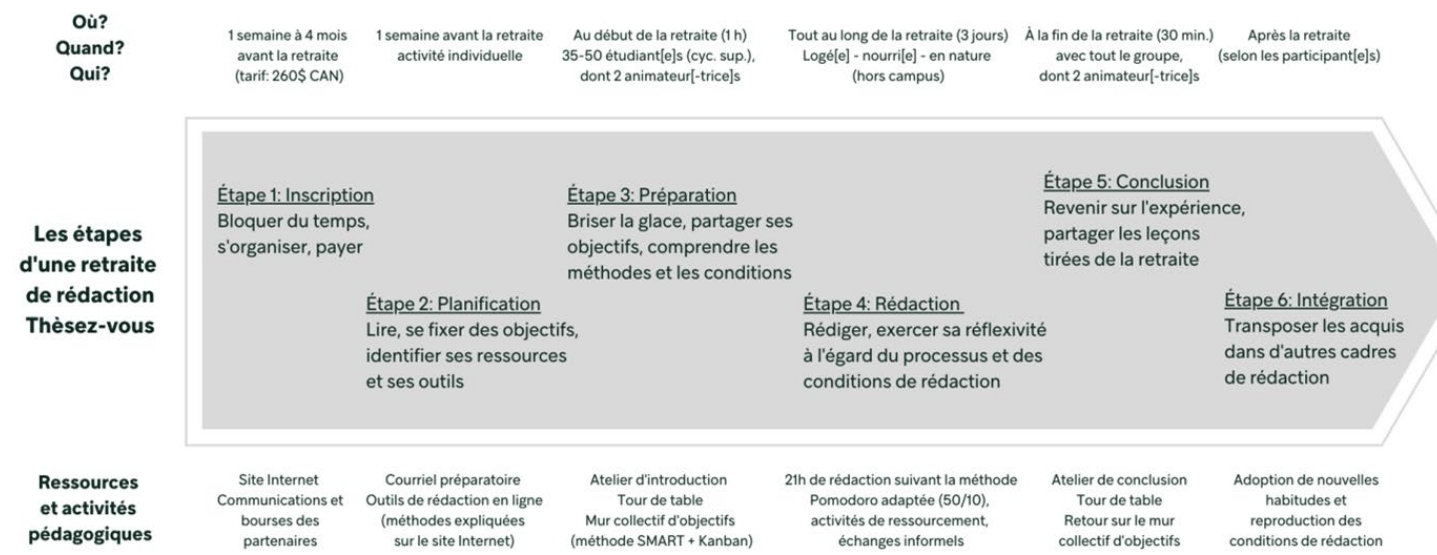

Si le champ de la recherche sur les retraites de rédaction est en pleine émergence dans le monde anglo-saxon, notamment au Royaume-Uni, en Australie et aux États-Unis, il est encore très peu documenté au Canada et au Québec, voire dans la francophonie en général. En outre, les résultats de ces recherches ont démontré que les retraites de rédaction améliorent la productivité scientifique (Kornhaber et al., 2016). Dans la plupart des recherches qui s'y intéressent, les retraites de rédaction décrites et évaluées ciblent essentiellement des médecins, des chercheur[-euse]s, des enseignant[e]s, des professionnel[le]s de la recherche ou des membres de facultés. Jusqu'à aujourd'hui, il semble qu'à l'exception des études de Papen et Thériault (2018), Stevenson (2020) et Stewart (2018), peu d'études ont examiné spécifiquement les retraites de rédaction destinées aux étudiant[e]s de cycles supérieurs, et aux doctorant[e]s plus particulièrement. 
Considérant ce qui précède, cet article a pour objectifs 1) de recenser les bénéfices des retraites de rédaction structurées tels que perçus par les doctorant[e]s en période de rédaction, et 2) d'identifier les conditions favorables à l'émergence de ces bénéfices.

\section{Les bénéfices des retraites de rédaction structurées et leurs conditions d'émergence}

La revue systématique des écrits réalisée par Kornhaber et al. (2016) fait état de travaux issus de 11 articles scientifiques qui démontrent que les retraites améliorent la productivité scientifique grâce à des bénéfices organisationnels, professionnels et personnels (Figure 2).

\section{Figure 2}

Schéma conceptuel des bénéfices des retraites de rédaction et de leurs thèmes clés

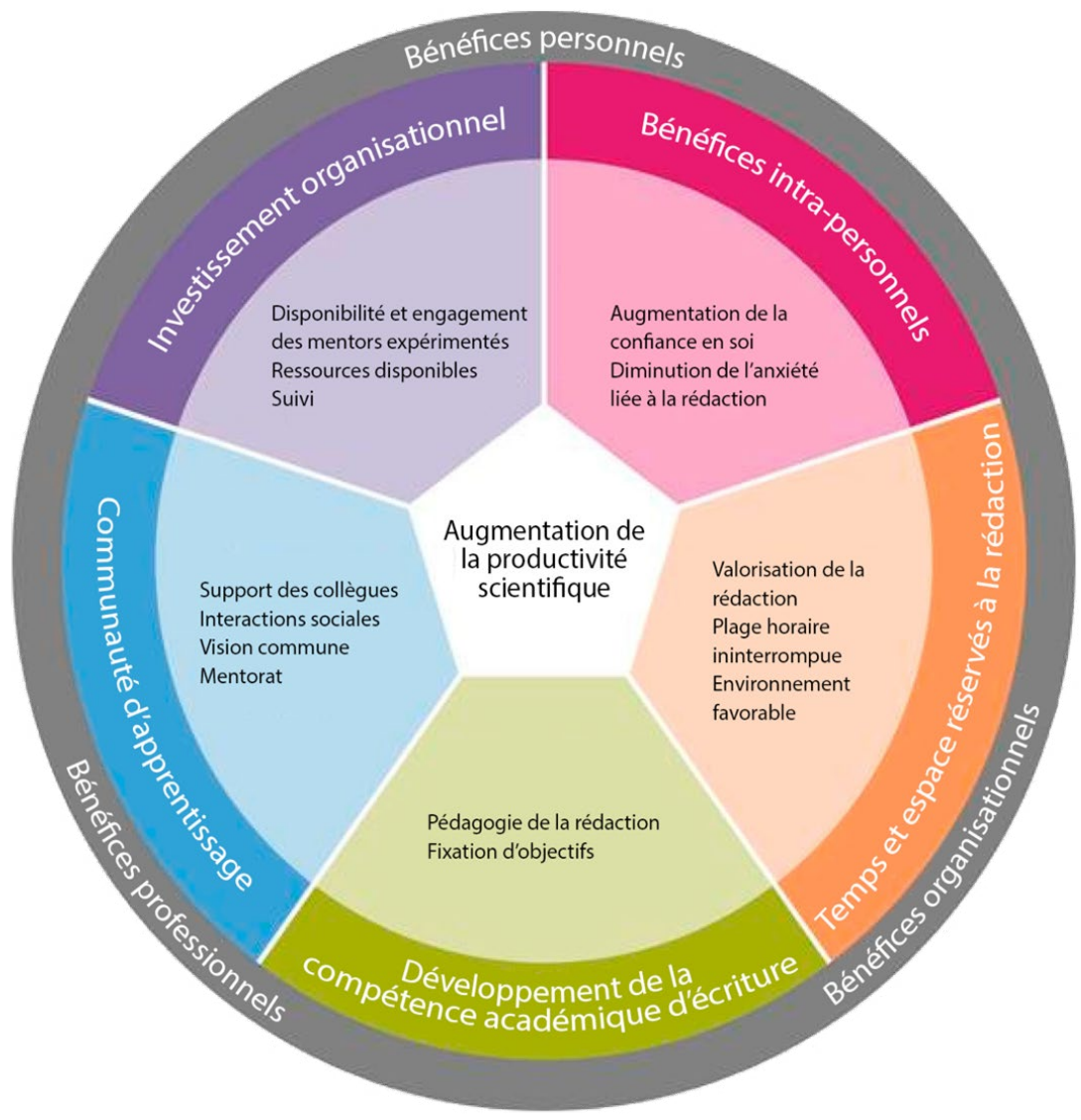

Note. Tirée de Kornhaber et al. (2016), traduction libre. 
Selon Kornhaber et al. (2016), pour qu'une retraite procure des bénéfices personnels, organisationnels et professionnels, elle doit :

- favoriser le développement d'une meilleure connaissance de soi, d'une confiance en soi et d'une motivation intrinsèque pour diminuer l'anxiété relative à la rédaction ;

- proposer un horaire prolongé et intensif sans distraction et un environnement confortable et sécurisant ;

- développer la compétence « savoir-écrire » en proposant des activités pédagogiques (cours, ateliers, jeux, modelage, écriture libre, etc.) et en insistant sur la fixation d'objectifs spécifiques et atteignables pour favoriser un sentiment d'accomplissement chez les participant[e]s ;

- favoriser une expérience rassembleuse en créant une communauté d'apprentissage ayant une vision commune, favorisant la collégialité, le mentorat et l'interaction avec les pairs ;

- S'assurer de la disponibilité et d'un engagement soutenu de la part de mentors expérimentés ainsi que prévoir suffisamment de ressources matérielles et financières pour mener à bien une telle activité.

En outre, la collégialité, du temps dédié à la rédaction, et la fixation d'objectifs personnels suivie d'une autoévaluation de ceux-ci contribuent aux bénéfices intrapersonnels que procure une retraite de rédaction.

Les principaux résultats des études recensées par Kornhaber et al. (2016) et les recherches subséquentes dans le domaine (Bullion et Brower, 2017 ; Maheux-Pelletier et al., 2019 ; Ratković et al., 2019) s'accordent pour dire que les retraites de rédaction structurées favorisent la productivité scientifique. Toutefois, peu mentionnent le caractère social et plaisant des retraites de rédaction. D'autres voient une incompatibilité entre le fait d'être axé sur la productivité scientifique et de vivre une expérience agréable. Par exemple, Bozalek (2017) propose de s'inspirer du slow scholarship en centrant la rédaction sur l'attention, le bienêtre, la réflexion et la qualité du travail produit plutôt que sur la production scientifique. Elle explique qu'une approche fondée sur la compétitivité, l'individualisme et la productivité entraine un sentiment de pression lié à la performance (Mountz et al., 2015). Ainsi, Bozalek (2017) suggère de faire la promotion d'expériences 
agréables en passant par les retraites de rédaction en tant qu'expériences collectives, inspirantes et créatives.

En Angleterre, Papen et Thériault (2018) ont étudié des retraites de rédaction qui avaient été organisées dans le cadre d'un programme d'études doctorales. Dès lors, 19 doctorant[e]s témoignent que la retraite a été plaisante, positive dans leur parcours et qu'elle a contribué à modifier positivement leur relation à la rédaction, et surtout, qu'elle a contribué à développer leur identité de rédacteur[-trice]. Également, dans sa thèse, Stewart (2018) s'est intéressée à des retraites pour doctorant[e]s aux États-Unis et rapporte des bénéfices au regard de la productivité scientifique, mais aussi en ce qui a trait à la motivation à écrire et à bâtir une communauté. Ces résultats démontrent l'importance de telles initiatives pour les doctorant[e]s et l'intérêt d'en proposer davantage (Papen et Thériault, 2018).

\section{Référents théoriques}

Prenant appui sur la recension des écrits de Kornhaber et al. (2016) afin de catégoriser les résultats, la présente étude repose également sur des référents théoriques issus de la théorie des systèmes écologiques (Bronfenbrenner, 1979) et du flow (Csikszentmihalyi, 1975) afin de mieux réfléchir aux conditions d'émergence des bénéfices liés aux retraites de rédaction. D'une part, la retraite de rédaction permet la création d'une communauté de pairs qui peut être analysée sous l'angle du microsystème (Bronfenbrenner, 1979), comme repris dans le modèle transactionnel et systémique de la rédaction de travaux universitaires de Kempenaar et Murray (2016). D'autre part, le concept de flow proposé par Csikszentmihalyi (1975) a été mobilisé lors de l'analyse des données qualitatives étant donné sa pertinence pour décrire les facteurs favorables à l'expérience optimale de rédaction vécue par les doctorant $[\mathrm{e}] \mathrm{s}$ lors des retraites.

\section{La théorie des systèmes écologiques : la communauté comme microsystème}

Bronfenbrenner (1979) a proposé cinq systèmes interconnectés pour mettre en lumière où l'individu se situe : le microsystème, le mésosystème, l'exosystème, le macrosystème et le chronosystème (pour refléter l'influence du temps). Au centre, l'individu s'inscrit 
dans un microsystème. Selon Kempenaar et Murray (2016), pour un universitaire, cela fait référence à l'environnement de proximité, soit l'environnement universitaire. Ces autrices soutiennent que les chercheur[-euse]s qui réussissent à rédiger de façon efficace se créent ou se positionnent dans des microsystèmes de soutien en travaillant avec des collègues qui valorisent la rédaction, en faisant partie de centres d'excellence et en s'entourant d'équipes et d'étudiant[e]s avec lesquel[le]s ils [elles] collaborent dans le cadre d'activités de recherche.

Bien que le modèle écologique de Bronfenbrenner (1979) ait été développé initialement pour conceptualiser le développement de l'enfant, les adultes et leurs pratiques d'écriture peuvent être considérés comme faisant partie d'un continuum d'apprentissage tout au long de leur vie. Ce faisant, tout comme Kempenaar et Murray (2016), nous estimons que cela rend ce modèle applicable au contexte universitaire, notamment aux doctorant[e]s et à la nature évolutive de leurs pratiques d'écriture.

\section{Le flow : la retraite de rédaction comme expérience optimale}

Csikszentmihalyi (1975) s'est penché sur le concept de flow dans le cadre de ses recherches sur la créativité et le bonheur, et l'a désigné comme une « expérience consciente, positive et complexe qui exprimait le sentiment de fluidité et de continuité ainsi que l'importante concentration que décrivaient les personnes interrogées lorsqu'elles exerçaient leur activité préférée » (Csikszentmihalyi et Bouffard, 2017, p. 65). Pour être dans le flow, une activité doit permettre de se sentir absorbé[e] par la pratique de cette activité (Fullagar et al., 2013). De plus, des conditions et une structure qui favorisent le flow sont nécessaires, notamment le fait d'avoir une cible claire, une concentration intense, un sentiment que la tâche entreprise est réalisable, mais qu'elle constitue un défi, un sentiment de contrôle sur ses actions et une perception d'intemporalité (Csikszentmihalyi et Csikszentmihalyi, 1988). Enfin, Kawabata et Mallett (2011) précisent que le flow peut être expérimenté plusieurs fois au cours d'une activité, et ce, même si celle-ci se prolonge sur une durée plus longue.

En fonction du contexte, de l'activité réalisée et de la présence de certaines conditions, le flow connait des fluctuations qui sont représentées dans la Figure 3 ci-après. 


\section{Figure 3}

La dynamique du flow

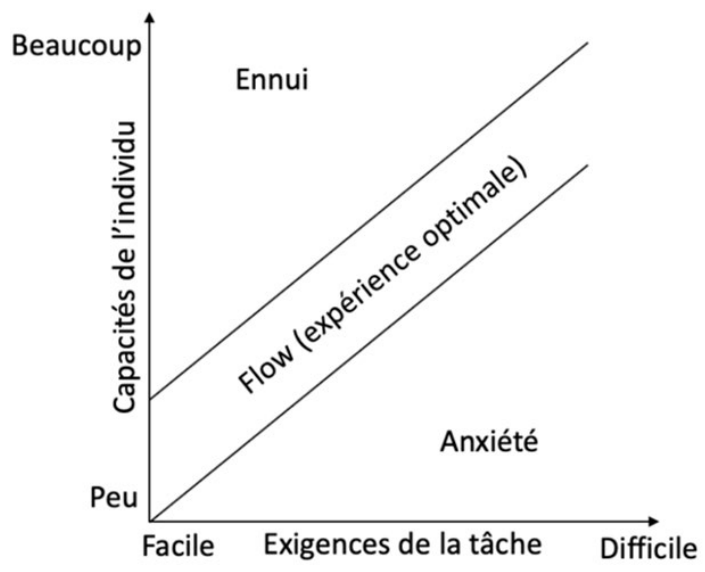

Note. Tiré de Csikszentmihalyi (1975).

Dans le contexte de rédaction, expérimenter le flow, soit une expérience optimale, implique de percevoir la tâche à réaliser comme un défi surmontable. Pour susciter cette expérience de flow, Csikszentmihalyi et Csikszentmihalyi (1988) mentionnent le fait de rassurer les participant[e]s sur ce qu'ils entreprennent, de les encourager à prendre des risques et à accepter leurs erreurs, de favoriser leur motivation intrinsèque ainsi que d'améliorer leur sentiment de compétence, leurs capacités de résolution de problèmes, leur autonomie, leur estime et leur confiance en soi.

\section{Méthodologie}

Les données présentées dans cet article ont été collectées dans le cadre d'une recherche visant à comprendre l'impact des retraites de rédaction Thèsez-vous suivant un protocole de recherche longitudinal (avant-après-suivis) avec groupe témoin (Tableau 1). Le groupe expérimental (GE) se compose de 70 doctorant[e]s ayant participé à une retraite de rédaction Thèsez-vous à l'hiver 2019, alors que le groupe contrôle (GC), de 140 doctorant[e]s n'ayant jamais pris part à des activités organisées par Thèsez-vous. 
Les doctorant $[\mathrm{e}] \mathrm{s}$ des deux groupes ont reçu un courriel les invitant à répondre à un questionnaire en ligne dans la semaine suivant la réception du courriel. Pour répondre aux critères d'éligibilité de l'étude, seul[e]s les participant[e]s inscrit[e]s dans un programme de doctorat au Québec et en Ontario et ayant terminé leur période de cours (c'est-à-dire, rendu[e]s au stade de rédaction de thèse) ont été retenu[e]s. Une approche mixte a été privilégiée étant donné la complémentarité des données qualitatives et quantitatives (Amyotte, 2011) en vue de comprendre les effets des retraites de rédaction sur les doctorant[e]s. Ainsi, 15 doctorant[e]s du GE et 15 doctorant[e]s du GC ont été interviewé[e]s à deux reprises, suivant le rythme des collectes de données quantitatives.

\section{Tableau 1}

Temps de collecte de données selon les volets de la recherche

\begin{tabular}{lcc}
\hline \multicolumn{1}{c}{ Groupe } & T1 & T2 \\
\hline Volet qualitatif & H2019 & \\
GC & H2019, une semaine après la retraite & 6 à 8 semaines après le T1 \\
GE & H2019 & \\
\hline Volet quantitatif & 2 à 8 semaines après le T1 \\
GC & H2019, une semaine avant la retraite & \\
GE &
\end{tabular}

\section{Le volet qualitatif}

Pour le volet qualitatif, les données ont été collectées auprès d'un sous-échantillon de 30 doctorant[e]s ayant répondu au questionnaire. Ceux [celles] du GE (hommes $=4$; femmes $=10$; non-binaire $=1$ ) provenaient de six universités à travers le Québec et réalisaient leur programme dans cinq domaines différents. Quant aux doctorant[e]s du $\mathrm{GC}$ (hommes $=6$; femmes $=9$ ), ils [elles] provenaient de sept universités à travers le Québec et réalisaient leur programme dans quatre domaines différents. Toutefois, comme l'objectif de cet article est de recenser les bénéfices des retraites et leurs conditions d'émergence, seules les données issues des entretiens du GE sont considérées. Deux entretiens semi-dirigés, d'une durée moyenne de quarante-cinq minutes, avaient pour objectif de faire émerger d'autres bénéfices en lien avec la participation à la retraite de 
rédaction Thèsez-vous, ainsi que d'explorer les fluctuations du flow de l'écriture vécues pendant celle-ci. Cet outil a fait l'objet d'un prétest en amont auprès de six doctorant[e]s. À la suite de la collecte des données, une fiche thématique a été bâtie et a servi à coder l'ensemble du corpus selon l'approche d'analyse du contenu manifeste de Van der Maren (2003), à l'aide du logiciel NVivo12. Un codage inverse a permis de s'assurer que chaque extrait codé appartenait bien au thème. Enfin, une validation interjuges a permis d'obtenir un pourcentage d'accord de $85 \%$ conformément aux critères établis par Cohen (1960). Afin d'identifier les participant[e]s aux entretiens cité[e]s dans cet article, tou[te]s ont reçu un pseudonyme alphanumérique dans lequel les deux premières lettres indiquent le groupe d'appartenance du [de la] participant[e] (GC ou GE), suivies d'un chiffre entre 1 et 15 désignant son identité.

\section{Le volet quantitatif}

La collecte des données quantitatives a été réalisée à l'aide d'un questionnaire en ligne. Les items de mesure ont été conçus à partir du modèle transactionnel et systémique de la rédaction de travaux universitaires de Kempenaar et Murray (2016). Les participant[e]s ont répondu en deux temps de mesure. Le GE était principalement composé de femmes $(76 \%$; hommes $=24 \%)$ et les participant[e]s de ce groupe avaient en moyenne 35 ans $(E ́ T=7.95)$. En ce qui a trait au GC, les participant[e]s étaient également en majorité des femmes $(77.1 \%$; hommes $=22.9 \%)$ et avaient en moyenne 32 ans $(E ́ T=7.27)$. Au T1, les doctorant[e]s du GE avaient complété une moyenne de 14.9 sessions d'études (ÉT= 8.97) dans le cadre de leur programme, tandis que ce nombre était de 10 sessions d'études $(E ́ T=5.02)$ pour les doctorant[e]s du GC.

Notons que l'ensemble des analyses ont été conduites avec les logiciels Mplus (version 7, Muthén et Muthén, 1998-2017) et IBM SPSS Statistics (version 25). Pour cet article, nous avons fait le choix de nous concentrer sur la conception de microsystème (ci-après nommé « communauté »). Les participant[e]s ont répondu à quatre items (par ex. : « Je sens que j'appartiens à une communauté d'étudiant[e]s en rédaction ») à l'aide d'une échelle de Likert en 7 points $(1=$ pas $d u$ tout en accord à $7=$ totalement en accord). Après avoir vérifié la distribution normale des données, nous avons constaté une cohérence interne acceptable ( $a=.79$ au T1 et .84 au T2). Par la suite, une analyse factorielle confirmatoire a été menée afin de confirmer l'existence de la conception de 
communauté et l'ajustement aux données du modèle au T1 et au T2. Pour ce faire, les indices d'ajustement suivants ont été utilisés : la statistique du khi carré $\left(\chi^{2}\right)$, le Root Mean Square Error of Approximation (RMSEA), le Standardized Root Mean Square Residual (SRMR), le Tucker-Lewis Index (TLI), et le Comparative Fit Index (CFI). Les analyses factorielles confirmatoires donnent les résultats suivants : au T1 $\left(\chi^{2}[2, n=2.17]\right.$, $p<.001 ; \mathrm{RMSEA}=.021[.00, .15] ; \mathrm{SRMR}=.017 ; \mathrm{CFI}=.999 ; \mathrm{TLI}=.997)$ et au T2 $\left(\chi^{2}[2, n=4.23], p<.001 ; \mathrm{RMSEA}=.097[.00, .23] ; \mathrm{SRMR}=.023 ; \mathrm{CFI}=.987 ; \mathrm{TLI}=\right.$ $.961)$. Au T1, 193 étudiant[e]s ont répondu au questionnaire $(\mathrm{GE}=55, \mathrm{GC}=138)$ et 119 étudiant $[\mathrm{e}] \mathrm{s}(\mathrm{GE}=52, \mathrm{GC}=67)$ au T2. Entre le $\mathrm{T} 1$ et le $\mathrm{T} 2$, nous avons eu une attrition de $38.34 \%$ des participant $[\mathrm{e}] \mathrm{s}(\mathrm{GE}=5.45 \%, \mathrm{GC}=51.45 \%)$. Ce taux d'attrition pourrait s'expliquer par le fait que, pour plusieurs participant[e]s, le T1 et le T2 ont pu sembler rapprochés dans le temps. Il est donc possible que certains d'entre eux [elles] aient omis de répondre au questionnaire du T2 croyant qu'il s'agissait d'un rappel du T1. Cela est d'autant plus plausible pour les participant[e]s du GC pour qui le T1 et le T2 n'étaient pas « délimités » par une retraite de rédaction. À partir des scores factoriels estimés en unités standardisées, un test- $t$ pour échantillons appariés a ensuite été réalisé au sein de chaque groupe (GE et GC) afin d'évaluer la différence entre le niveau du sentiment de communauté des participant[e]s au T1 et au T2. Enfin, deux tests-t pour échantillons indépendants ont été réalisés pour comparer le niveau du sentiment de communauté du GE et du GC au T1 et au T2.

\section{Résultats}

Nous avons organisé la présentation des résultats de recherche autour des bénéfices des retraites de rédaction structurées et des conditions qui facilitent l'émergence de ces bénéfices. L'approche privilégiée dans cet article en est une de compréhension et d'interprétation. En ce sens, les données issues du volet qualitatif sont prédominantes, et complétées par quelques résultats quantitatifs qui permettent d'étendre la réflexion au-delà de l'échantillon interviewé. 


\section{Les bénéfices des retraites structurées Thèsez-vous}

À l'issue de l'analyse des entretiens, trois bénéfices ressortent : la retraite a permis de 1) favoriser la rédaction, 2) légitimer la rédaction et 3) vivre une expérience plaisante.

D'abord, les retraites de rédaction Thèsez-vous sont l'occasion de favoriser la rédaction, car elles permettent de réaliser une grande quantité de travail en peu de temps (43 extraits). Plusieurs doctorant[e]s ont ainsi réussi à rédiger « une section » entière (GE10), voire « un chapitre complet» (GE7). De plus, la participation à une retraite semble permettre aux participant[e]s d'en apprendre plus sur les conditions optimales de rédaction (32 extraits), en rapportant avoir découvert des techniques d'organisation temporelle. Toutefois, il ressort des entretiens que les participant[e]s éprouvent de la difficulté à transposer seul[e]s ces apprentissages : « Je fais partie des gens qui n'arrivent peut-être pas complètement à tout transposer. J'ai vraiment besoin de la retraite en soi, [qui] est l'outil suprême pour moi » (GE1). Il apparait ainsi que les retraites sont essentielles, car elles permettent non seulement aux étudiant[e]s de découvrir des techniques appropriées, mais également de les mettre en pratique dans un environnement et un encadrement favorables. Or, si certain[e]s réussissent à reproduire ces conditions dans leur quotidien pour un certain temps, ces pratiques efficaces semblent s'estomper au fil des jours. Ainsi, les participant[e]s conviennent qu'ils ont besoin de revenir en retraite pour se rappeler ces façons de faire :

Je pense que c'est comme une piqure de rappel, car on se laisse envahir par plein d'autres choses. Tu as la méthode, tu l'appliques, ça marche, tu es content. Mais petit à petit, les autres choses commencent à grignoter [du temps] et les plages horaires que tu essaies de maintenir coute que coute s'espacent. Tu te fais grignoter. Pour moi, il me faut des retraites assez régulièrement pour que ça continue sur cette lancée (GE12).

Ensuite, légitimer la rédaction représente un défi de taille dans la mesure où cette tâche est rarement considérée comme prioritaire aux yeux des doctorant[e]s et de leur entourage professionnel et personnel. Étant donné la difficulté à prioriser la rédaction au profit d'autres tâches, les participant[e]s utilisent la retraite Thèsez-vous comme stratégie pour rendre légitime leur rédaction (18 extraits). Les bénéfices associés à cette légitimité s'articulent à deux niveaux. Premièrement, cela rend la tâche plus « sérieuse » 
et importante aux yeux de l'entourage. Deuxièmement, l'expérience de retraite est gratifiante, car les participant[e]s démontrent un intérêt et valorisent les travaux de chacun[e] : «C'est ça que je vais chercher [en retraite] : des gens qui s'intéressent vraiment et sincèrement au projet des autres » (GE13).

Enfin, nombre de participant[e]s soulignent qu'un des principaux bénéfices des retraites Thèsez-vous est de faire de la rédaction une expérience plaisante (28 extraits). Soulignons que l'ensemble des doctorant[e]s questionné[e]s lors des entrevues mentionnent avoir apprécié leur retraite. « Content[e]s » et « motivé[e]s », ils [elles] caractérisent la retraite comme une expérience « agréable », « géniale », « trippante », « plaisante », « le fun », « fantastique », etc. Lorsque questionné[e]s sur la nature de cette expérience plaisante, ils [elles] expliquent qu'en retraite, les périodes de rédaction leur permettent d'atteindre un flow (l'expérience optimale) qu'ils[elles] vivent rarement ailleurs. Un doctorant explique :

Le flow, $[. .$.$] c'est comme une période dans laquelle tu es dans le moment présent$ [...] où tu es juste concentré sur la tâche et le monde extérieur est comme moins envahissant, moins présent dans ton esprit. Tu es plus efficace [...] pour avancer dans ce que tu fais. (GE15)

En somme, les retraites de rédaction sont bénéfiques à la persévérance en favorisant la rédaction de sections substantielles, en légitimant, voire en valorisant cette tâche, ainsi qu'en permettant de vivre une expérience plaisante qui contraste avec leur rapport habituel à la rédaction. La Figure 4 présente ces bénéfices en parallèle des conditions qui facilitent leur émergence. 


\section{Figure 4}

Les bénéfices des retraites de rédaction Thèsez-vous

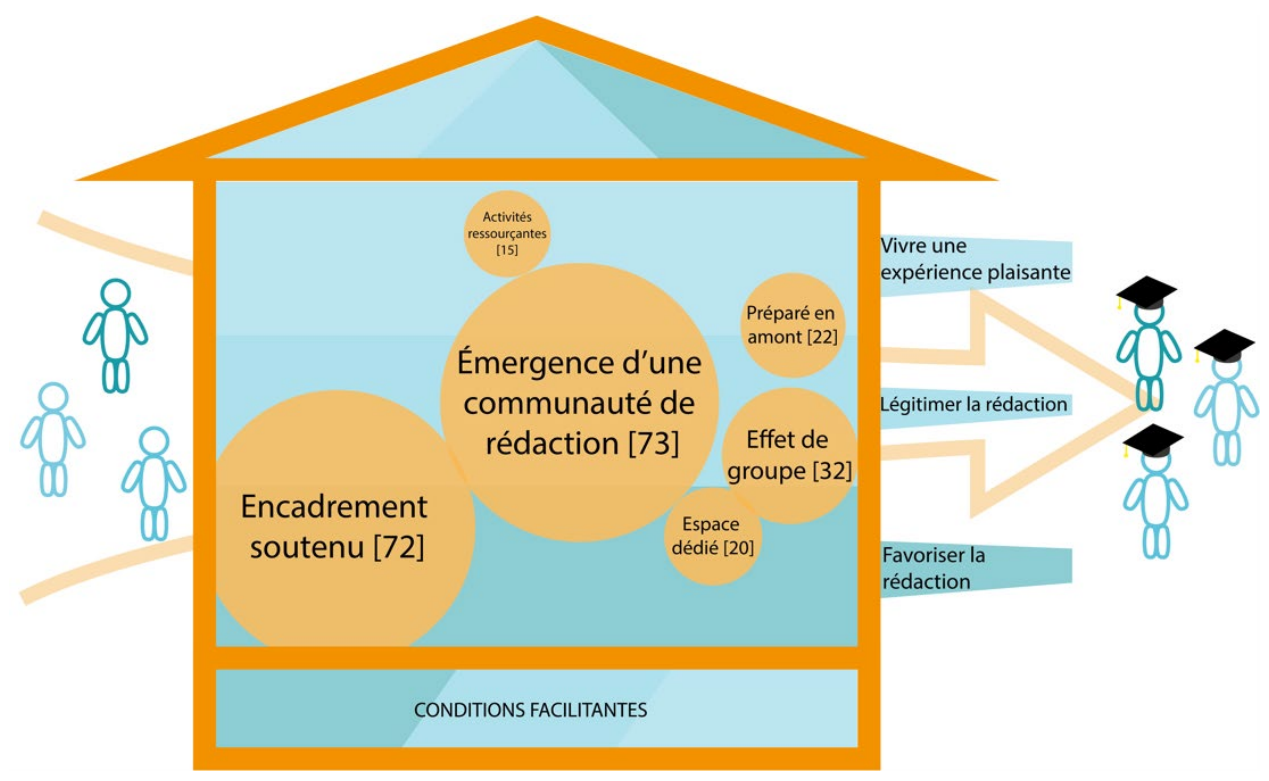

\section{Les conditions facilitantes}

Au-delà des bénéfices énoncés (favoriser la rédaction, légitimer la rédaction et permettre de vivre une expérience plaisante), les résultats ont permis d'identifier les conditions favorables associées à ces bénéfices à partir de l'expérience des doctorant[e]s interrogé[e]s.

L'importance de la communauté. Les participant[e]s s'inscrivent individuellement en retraite pour avancer leur projet et ne se connaissent pas forcément puisqu'ils[elles] proviennent de diverses disciplines et universités. Il est donc primordial de permettre le développement d'un sentiment de communauté dès l'atelier d'introduction de la retraite. La communauté créée et entretenue lors des retraites est considérée comme importante par l'ensemble des doctorant[e]s qui ont participé aux entretiens (15/15: 73 extraits), la communauté devenant alors un microsystème de référence. Selon les participant[e]s, le développement de ce sentiment de communauté découle de trois conditions mises en place lors des retraites de rédaction : le partage d'une réalité commune, la socialisation organisée et la culture de bienveillance. 
Premièrement, se regrouper entre individus qui rédigent semble dès le départ faire naitre un sentiment d'appartenance à la communauté par le partage d'une réalité commune. En effet, les participant[e]s mentionnent l'intérêt de s'entourer d'étudiant[e]s ayant des préoccupations similaires. Qu'il soit question du sentiment d'isolement ressenti ou de leur relation parfois houleuse avec leur direction de thèse, ils [elles] apprécient et se sentent soulagé[e]s de pouvoir partager leur vécu avec des pairs qui comprennent ce qu'implique un parcours doctoral. Deuxièmement, les moments structurés de socialisation consolident ce sentiment de communauté. Les participant[e]s apprécient que les animateur[-trice]s encouragent les échanges informels lors de pauses planifiées, ce qui a pour effet de valoriser la socialisation sans nuire à la productivité et à la concentration. Ces moments d'échanges sont l'occasion de « créer des liens », ce qui est vu comme une « récompense » à la suite des efforts accomplis en termes d'objectifs de rédaction. Ils [elles] ne vont pas seulement chercher à travailler, mais bénéficient également des relations qu'ils[elles] créent avec des gens « qui s'intéressent sincèrement aux projets des autres » (GE13). Étant donné la diversité de profils des participant[e]s, les échanges sont teintés d'ouverture et de curiosité à l'égard de différents champs de recherche et projets : « même si ça ne concerne pas directement ma méthodologie ou ma façon de travailler, ça m'a ouvert à questionner ma propre façon d'envisager la rédaction » (GE1). Troisièmement, les animateur[ trice]s de la retraite veillent à ce qu'elle soit un milieu sécurisant et bienveillant. Peu importe l'adjectif utilisé par les participant[e] s pour qualifier l'ambiance : « chaleureuse », « amicale », « collégiale », « conviviale », « collective », « communautaire » ou « solidaire », il semble que la retraite permette de se sentir « entouré[e]s » et « supporté[e]s ». Les participant[e]s rapportent que la compétition ressentie entre doctorant[e]s et le sentiment d'imposteur sont absents en retraite. Au contraire, ils [elles] sont encouragé[e]s par les réussites des autres et l'atteinte de leurs objectifs, ce qui témoigne du développement d'une sensibilité au succès collectif. Cette bienveillance semble alléger la situation parfois douloureuse et « lourde » vécue par certain[e]s participant[e]s. Puisque l'isolement pèse pour certain[e]s, les échanges apportent un « baume » dans leur parcours doctoral : « j'ai même vu une des participantes qui était un peu épuisée et qui venait de faire un objectif difficile qui a comme pleuré. Les gens étaient là pour la supporter » (GE6).

Les résultats issus des analyses quantitatives soutiennent l'importance accordée au sentiment de communauté pour les étudiant[e]s qui participent aux retraites de rédaction 
Thèsez-vous en comparaison avec les étudiant[e]s du GC. En effet, les participant[e] s du GE ont un score factoriel de .790 au T1 et de .705 au T2, alors que ceux [celles] du GC en ont un de .141 au T1 et de .637 au T2. Afin de vérifier si ces différences sont significatives, des tests- $t$ indépendants ont été menés, montrant une différence statistiquement significative entre le sentiment de communauté du GE et celui du GC au T1 $(t[189]=4.39, p<.0001,95 \%$ CI $[.465,1.303], d=.69)$ et au T2 $(t[115]=4.93$, $p<.0001,95 \%$ CI $[.796,1.865], d=.92)$. Ainsi, les participant[e]s du GE présentent significativement un plus fort sentiment de communauté aux deux temps de mesure. Ensuite, des tests- $t$ appariés ont été menés afin de vérifier les différences intragroupes entre le T1 et le T2. Il s'avère que le sentiment de communauté des participant[e]s du GE est le même au T1 et au T2 $(t[35]=.514, p=.610,95 \%$ CI $[.250, .420], d=0.0)$. Cela s'explique notamment par le fait que la plupart des étudiant[e]s du GE ayant rempli le questionnaire n'en étaient pas à leur première retraite de rédaction Thèsez-vous. De plus, il est possible que les étudiant[e]s qui s'inscrivent à une retraite reconnaissent leur besoin de briser l'isolement ainsi que ses bénéfices sur la productivité et la persévérance. À l'inverse, les résultats indiquent qu'une différence existe pour les participant[e]s du GC, chez qui une diminution du sentiment de communauté a été observée entre le T1 et le T2 $(t[64]=4.98, p<.0001,95 \%$ CI [.297, .695], $d=.61)$. Cette diminution au sein du GC s'explique notamment par l'effet potentiel du questionnaire à l'égard d'une prise de conscience sur la place accordée à la communauté dans leur rapport à la rédaction et l'absence d'expérience collective depuis le T1.

L'encadrement soutenu. La rédaction est une tâche ardue qui nécessite une concentration soutenue et profonde. Les participant[e]s soulignent que l'encadrement offert lors des retraites Thèsez-vous, particulièrement la technique de gestion, favorise cette concentration (72 extraits). Cette technique (adaptation de la technique Pomodoro à un cadre de rédaction collective) consiste à rédiger pendant une période de 50 minutes, suivie d'une pause de 10 minutes. Après une séquence de quatre pomodoros, une période de ressourcement plus longue est prévue. Comme les retraites se déroulent sur plusieurs jours, les participant[e]s mentionnent qu'il serait impossible de maintenir un tel rythme de rédaction sans ces moments de pause. Ceux-ci, « imposés » par les animateur[-trice]s, augmentent en quelque sorte la qualité de la rédaction produite. De plus, le fait d'être pris 
en charge permet aux participant[e]s de se concentrer sur leur rédaction, ce qui améliore leur expérience et diminue leur charge mentale :

C'était très bien pour mon écriture de juste focusser sur ce que j'avais à faire. Et aussi, j'ai deux jeunes enfants [...], je n'avais pas besoin de penser aux besoins des autres, juste aux miens, et même les miens étaient tellement simplifiés ! Je vivais un rêve. Tous mes repas étaient préparés. C'était vraiment une simplicité qui était conductible pour avancer et me concentrer. (GE3)

Ces séquences de temps encadré contribuent à la productivité des étudiant[e] s par le silence imposé et l'ambiance de concentration collective, mais également à la légitimité de la tâche en protégeant concrètement du temps dédié à la rédaction.

L'effet de groupe. Une des conditions facilitantes des retraites Thèsez-vous est ce que l'on peut appeler l'effet de groupe (32 extraits) qui s'instaure au fil des périodes de travail. En effet, les participant[e]s rapportent être d'autant plus concentré[e]s à leur propre tâche lorsqu'ils[elles] observent les autres progresser dans leur rédaction. Bien qu'ils[elles] aient de la difficulté à décrire ce qui augmente tant leur productivité, ils [elles] témoignent de la présence d'une certaine « force » et d'une « énergie » qui se développent tout au long de la retraite. Les doctorant[e]s expliquent que cette énergie les entraine à ne pas abandonner et à persévérer dans la rédaction :

Je pense qu'il y a un aspect aussi de voir l'effort de groupe. [...] Pas de voir sur quoi [ils ou elles] travaillent, mais de voir que tout le monde travaille. Ce qui fait qu'un moment donné, tu ouvres Facebook puis là tu fais : c'est clair qu'il y a des gens qui voient mon écran en ce moment... Cela ne doit pas les motiver non plus, donc je vais juste le fermer, puis je retourne à mes affaires. (GE7)

Cet effet de groupe est perçu comme une pression sociale saine qui s'établit à deux niveaux. Premièrement, le fait de voir les autres à la tâche encourage chaque étudiant[e] à persévérer. Deuxièmement, ils [elles] ont le souci de ne pas distraire ou déranger leurs pairs et évitent donc des comportements associés à la procrastination, qu'il soit question d'aller sur les réseaux sociaux ou de se lever hors des moments prévus pour les pauses. 
La préparation en amont. La participation à une retraite implique une préparation en amont (22 extraits). Dans un courriel envoyé quelques semaines avant la retraite, son fonctionnement est expliqué, en plus de faire valoir les avantages que pourrait produire une préparation adéquate. Un document explicatif invite les participant[e]s à préparer leur matériel et à faire leurs lectures en amont, mais aussi à réfléchir et à prévoir leurs objectifs de rédaction pour chaque plage identifiée dans l'horaire. Lorsqu'un participant a été questionné sur ce qui l'a amené à débloquer lors de la retraite, sa réponse était sans équivoque :

C'est la préparation avant une retraite en fait. Quand je m'en vais en retraite, je suis obligé de préparer mes objectifs de façon beaucoup plus claire. Et ça, c'est souvent un bout qui peut être plus difficile des fois. [...] Ça change tout de se préparer ! (GE1)

Cette condition est rapportée par plusieurs comme étant favorable à la réussite de leur retraite, puisqu'ils[elles] sont amené[e]s à amorcer leur démarche au moyen de l'identification d'objectifs de rédaction.

Un espace dédié. Les participant[e]s utilisent des qualificatifs évocateurs pour expliquer leur appréciation des différents lieux (20 extraits) dans lesquels prennent place les retraites Thèsez-vous : un site « enchanteur », « en nature », pourvu d'une « belle vue ». Ils [Elles] disent notamment apprécier le « silence » et l'immersion dans la nature, que ce soit la proximité avec les animaux présents sur certains sites d'hébergement ou le paysage « paisible et beau ». Certain[e]s disent que leur séjour s'apparente à des « vacances », bien qu'ils[elles] soient en mode « travail», puisqu'ils[elles] sont géographiquement éloigné[e]s de leur résidence et de leur quotidien pour rédiger dans un lieu dédié à la rédaction, « loin de toutes distractions ». Un participant compare sa rédaction maison à celle en retraite :

Un espace qui est réservé et dédié au travail [...] c'est vraiment un avantage. J'ai essayé de faire [...] des périodes de rédaction avec mes amis, chez nous [...], mais ça n'a pas été aussi efficace. De un, parce que je commence à parler à mes amis [...], mais aussi parce que ce n'est pas un espace qui est dédié à [la rédaction]. À 
la retraite, dans la grande pièce, où tout le monde était, j'ai associé dans ma tête : ici, on rédige et that's it!. Je pense que c'est ça qui m'a le plus focussé. (GE15)

Des activités qui ressourcent. Lors des retraites, des activités en plein air ou ludiques sont proposées aux participant[e]s. Ces dernier[-ère]s ne voient que des avantages à y participer (15 extraits). Plusieurs d'entre eux [elles] soulignent le clivage entre une journée de rédaction normale et une journée en retraite Thèsez-vous : « Sept ou huit [pomodoros] [...] dans une même journée, c'est plus difficile, c'est énorme! Alors qu'en [retraite], faire huit ou neuf [pomodoros] par jour ça se fait, car il y a des [activités] qui ponctuent »(GE1). Les répondant[e]s apprécient les activités proposées, comme les marches en plein air, la période de yoga, les jeux de société, etc. En effet, ces activités permettent de « décrocher » de manière à mieux se remettre à la rédaction par la suite.

\section{Discussion et pistes de recherches futures}

Cette étude entendait recenser les bénéfices des retraites de rédaction structurées pour les doctorant[e]s en période de rédaction et les conditions qui en facilitent l'émergence. Les résultats ont révélé trois principaux bénéfices à la participation à une retraite de rédaction ainsi que des conditions à mettre en place pour en favoriser l'émergence. Plusieurs études ont déjà soulevé les avantages perçus des retraites de rédaction, notamment en lien avec la productivité scientifique auprès de différentes populations universitaires (Kornhaber et al., 2016). Nos résultats confirment ces avantages auprès d'une population de doctorant[e]s, tout en apportant un nouvel éclairage quant au rôle que peuvent jouer les retraites de rédaction sur la légitimité du travail doctoral et l'importance de l'ancrer dans une approche collective.

\section{Des conditions pour une retraite de rédaction efficace : rédaction préparée, structurée et collective}

Une première condition est la préparation et l'organisation des participant[e]s en amont. Cette condition est d'ailleurs soulevée par Kornhaber et al. (2016) comme un bénéfice organisationnel qui mériterait d'être davantage étudié. D'une part, comme le soulève 
Stevenson (2020), nous concluons que la valorisation du bienêtre devrait être à la base de la planification d'une retraite de rédaction structurée par les organisateur[ trice]s. D'autre part, si quelques études ont traité de la préparation de la retraite par des organisateur[ trice]s, peu font état des apports de la préparation des participant[e]s à une retraite. Le modèle étudié dans notre recherche invite les participant[e]s à établir des objectifs de rédaction et à préparer leurs lectures quelques jours à l'avance. Cette façon de faire s'avère positive, puisqu'elle permet aux participant[e]s de profiter au maximum du temps de rédaction. À cet égard, Papen et Thériault (2018) rapportaient que le manque de préparation pouvait limiter la productivité et la qualité en induisant de la fatigue plutôt que du plaisir. Ainsi, les doctorant[e]s, étant des rédacteur[-trice]s novices, tirent d'autant plus profit d'une préparation adéquate avant de participer à une retraite, afin qu'elle soit plus productive et agréable.

Une autre condition à respecter est la mise en place de techniques et de stratégies structurantes qui se traduisent par un encadrement soutenu. D'une part, Kornhaber et al. (2016) soutiennent que l'encadrement du temps permet aux participant[e]s de garder un momentum d'écriture, sans toutefois spécifier la nature de cet encadrement. Notre étude offre cette précision en pointant que l'utilisation d'une version adaptée de la technique Pomodoro favorise une concentration soutenue (flow) tout en accordant des temps de pause et de socialisation. D'autre part, les participant[e]s expérimentent et s'approprient une méthode de fixation d'objectifs et utilisent collectivement la méthode Kanban, à l'aide de Post-it, afin d'apprécier leur progression et celle de leurs pairs. Ces données soutiennent celles obtenues par Grant (2006) qui illustrent aussi que les retraites favorisent l'adoption de nouveaux rituels, des habitudes, des habiletés ou des stratégies pour appréhender plus sereinement et efficacement la rédaction scientifique. Néanmoins, notre étude met en lumière que ces nouvelles habitudes de rédaction sont difficiles à transposer dans un cadre individuel de rédaction ou à maintenir dans le temps. En ce sens, plusieurs doctorant[e]s expriment le besoin de participer plus d'une fois à une retraite de rédaction.

Une dernière condition favorisant la productivité est l'émergence d'une énergie qui se développe lors des périodes de rédaction collective. Cet effet de groupe entraine les doctorant[e]s dans une forme de flow (Csikszentmihalyi et Bouffard, 2017 ; Janke et al., 2018) qui les amène à limiter la procrastination et à redoubler d'ardeur dans leur propre rédaction en raison d'une pression sociale qualifiée de positive. Ces constats vont 
dans le même sens que d'autres études réalisées auprès d'étudiant[e]s qui participent à des retraites structurées (Aitchison et Lee, 2006 ; Dwyer et al., 2012 ; Lee et Boud, 2003 ; Papen et Thériault, 2018), un élément toutefois absent des résultats de recherche s'intéressant aux retraites de rédaction pour professeur[e]s-chercheur[-euse]s. De futures recherches pourraient s'intéresser à l'effet de groupe et à ses retombées sur la productivité scientifique d'autres populations qui prennent part à des retraites de rédaction structurées.

\section{La retraite de rédaction structurée met en lumière l'importance de rendre le travail doctoral légitime et d'offrir un cadre protecteur}

Les résultats soulignent que la participation à une retraite de rédaction structurée permet aux doctorant $[\mathrm{e}] \mathrm{s}$ de légitimer leur rédaction. La mise en place d'un espace dédié à la rédaction est l'une des conditions à respecter pour y arriver. Comme identifié dans plusieurs recherches antérieures, se retrouver dans un centre de villégiature, loin de toutes distractions, permet d'offrir un espace-temps protégé. En effet, sur les 11 études recensées par Kornhaber et al. (2016), 10 rapportaient l'apport des retraites en tant que temps et espace dédiés. Cette condition permet non seulement aux doctorant[e]s de légitimer la rédaction à leurs propres yeux, mais également aux yeux de leur entourage. En comparaison avec les études concernant des professeur[e]s-chercheur[-euse]s, nos résultats permettent de souligner que la légitimité du travail doctoral est davantage remise en question et dévalorisée par l'entourage des doctorant[e]s. Des recherches futures devraient permettre de mieux comprendre pourquoi cette activité n'est pas reconnue comme un travail à part entière.

\section{La retraite de rédaction permet de vivre la rédaction dans une perspective collective et bienveillante plutôt qu'individuelle et compétitive}

Bien que la productivité soit un bénéfice important aux yeux des participant[e]s de notre étude, le développement d'un sentiment d'appartenance envers une communauté est un bénéfice particulièrement valorisé. Nos résultats indiquent que le fait de vivre une expérience de rédaction avec des étudiant[e]s qui partagent une même réalité est non seulement favorable à la productivité, mais contribue à en faire une expérience 
plaisante et optimale. La recherche de Mayrath (2008), mettant au jour les conditions de rédaction pour la productivité scientifique, révélait la valeur de la rédaction collective, plus précisément, collaborative sur un même texte. Notre étude apporte un nouvel éclairage à ces résultats, puisqu'elle démontre que la rédaction individuelle, mais en compagnie d'autres rédacteur[-trice]s est également bénéfique. Ces résultats confirment la pertinence de conceptualiser la rédaction dans une approche écologique, comme le proposent Bronfenbrenner (1979) et Kempenaar et Murray (2016), voire à transformer les représentations de la rédaction véhiculées dans le monde universitaire afin d'en faire une activité collective plutôt qu'une tâche individuelle. Dix des onze articles recensés par Kornhaber et al. (2016) identifient le phénomène d'adhésion à une communauté pendant une retraite de rédaction. Comme les doctorant[e]s ressentent un fort sentiment d'isolement lors de leur période de rédaction (Levecque et al., 2017), la formation d'une communauté de rédacteur[-trice]s pourrait être perçue comme un facteur de protection contre le sentiment d'abandon, en permettant de vivre de la solidarité et du plaisir au fil du parcours doctoral. D'ailleurs, ces constats vont dans le même sens que ceux énoncés par Papen et Thériault (2018) quant à l'importance des émotions positives et du plaisir que génère la participation à une retraite de rédaction, ainsi qu'à ceux de Stevenson (2020) en ce qui concerne le bienêtre. Par ailleurs, nos résultats mettent en lumière l'ambiance exempte de compétitivité qui est mise en place lors des retraites. Le fait que les doctorant[e]s proviennent de différentes disciplines et universités contribue probablement à la mise en place de cette bienveillance. En effet, Wardale et ses collègues (2015) avaient remarqué que la composition multidisciplinaire d'un groupe est avantageuse pour diminuer la compétitivité.

\section{Limites et retombées de la recherche}

L'étude menée comporte certaines limites qui doivent être reconnues et qui pourront orienter les recherches futures. Comme première limite, il aurait été intéressant d'inclure des mesures sur la date d'adhésion des participant[e]s à Thèsez-vous et sur leur nombre de participations aux activités de rédaction collective, puisque ces variables pourraient modérer, voire médier le sentiment de communauté. Une deuxième limite est liée à la méthode d'échantillonnage du volet qualitatif. Il est possible de croire 
que les participant[e]s ayant davantage apprécié la retraite soient ceux [celles] qui se sont porté[e]s volontaires pour les entretiens. Pour cette raison, les résultats quant à l'unanimité de l'appréciation positive des retraites devraient être nuancés. Malgré ces limites, cette recherche apporte des connaissances empiriques pertinentes, notamment en suggérant que les retraites de rédaction structurées comportent des bénéfices importants pour les doctorant[e]s. Sur cette base, il apparait que l'apport de la communauté de rédacteur[-trice]s qui se crée en retraite gagnerait à être mieux connu des étudiant[e]s et valorisé par les directions de recherche et les programmes d'études. De plus, nos résultats soulignent l'importance de considérer aussi les conditions facilitantes, des éléments susceptibles de moduler les bénéfices d'une retraite de rédaction pour des étudiant[e]s aux cycles supérieurs.

\section{Conclusion}

Cette recherche s'est attardée à décrire les bénéfices des retraites de rédaction Thèsezvous et les conditions facilitantes pour une expérience optimale. L'originalité de la recherche réside dans le fait d'étudier le potentiel et les limites des retraites sur le bienêtre et la productivité des doctorant[e]s et d'amener à réfléchir plus largement aux conditions qui favorisent la productivité, la légitimité et la place accordée à la communauté.

Les résultats ont non seulement mis en lumière l'apport de ces retraites en termes de productivité scientifique, mais aussi en ce qui a trait au sentiment de légitimité et de plaisir. Cette étude a été l'occasion de cerner les conditions à mettre en place pour favoriser l'émergence de ces bénéfices : 1) en créant une communauté de rédacteur[trice]s ; 2) en offrant un encadrement soutenu ; 3) en insufflant un effet de groupe ; 4) en proposant aux étudiant $[\mathrm{e}] \mathrm{s}$ de se préparer en amont ; 5) en ayant un espace dédié à la rédaction et 6) en offrant des activités favorisant le ressourcement. Qui plus est, on recense très peu de retombées négatives, ce qui met en relief le consensus concernant les bénéfices reliés à la participation à une retraite de rédaction structurée pour les étudiant[e] s, et ce, malgré les nuances mentionnées dans les limites de l'étude. Dans l'ensemble, les résultats révèlent qu'il est en effet possible de mettre en place des conditions qui favorisent la rédaction lors du parcours doctoral. De tels constats pourraient orienter le curriculum des programmes de doctorat afin qu'ils incluent des crédits pour la 
participation à une retraite de rédaction structurée. Sachant que les universités et les directions de recherche ont à cœur la réussite et le bienêtre de leurs étudiant[e] $\mathrm{s}$, elles doivent également remettre en question la productivité scientifique axée sur la performance et s'attarder à la socialisation dont les doctorant[e]s ont aussi besoin en période de rédaction. 


\section{Références}

Aitchison, C. et Guerin, C. (2014). Writing groups, pedagogy, theory and practice: An introduction. Dans C. Aitchison et C. Guerin (dir.), Writing groups for doctoral education and beyond: Innovations in practice and theory (p. 3-17). Routledge.

Aitchison, C. et Lee, A. (2006). Research writing: Problems and pedagogies. Teaching in Higher Education, 11(3), 265-278. https://doi.org/10.1080/13562510600680574

Amyotte, L. (2011). Méthodes quantitatives : applications à la recherche en sciences humaines (3e éd.). ERPI.

Bozalek, V. (2017). Slow scholarship in writing retreats: a diffractive methodology for response-able pedagogies. South African Journal of Higher Education, 31(2), 40-57. https://doi.org/10.20853/31-2-1344

Bronfenbrenner, U. (1979). The ecology of human development. Harvard University Press.

Bullion, J. W. et Brower, S. M. (2017). Enhancing the research and publication efforts of health sciences librarians via an academic writing retreat. Journal of the Medical Library Association, 105(4), 394-408. https://doi.org/10.5195/jmla.2017.320

Cohen, J. (1960). A coefficient of agreement for nominal scales. Educational and Psychological Measurement, 20(1), 37-46. https://doi. org/10.1177/001316446002000104

Csikszentmihalyi, M. (1975). Beyond boredom and anxiety: Experiencing flow in work and play. Jossey-Bass.

Csikszentmihalyi, M. et Bouffard, L. (2017). Le point sur le flow. Revue québécoise de psychologie, 38(1), 65-81. https://doi.org/10.7202/1040070ar

Csikszentmihalyi, M. et Csikszentmihalyi, I. S. (dir.). (1988). Optimal experience: Psychological studies of flow in consciousness. Cambridge University Press.

Denis, C. (2020). Pratiques d'encadrement à la recherche au doctorat en contexte francophone nord-américain : à la découverte de balises [Thèse de doctorat, Université de Sherbrooke]. Savoirs UdeS. https://savoirs.usherbrooke.ca/ handle/11143/17357 
Dwyer, A., Lewis, B., McDonald, F. et Burns, M. (2012). It's always a pleasure: Exploring productivity and pleasure in a writing group for early career academics. Studies in Continuing Education, 34(2), 129-144. https://doi.org/10.1080/01580 $\underline{37 X .2011 .580734}$

Fullagar, C. J., Knight, P. A. et Sovern, H. S. (2013). Challenge/skill balance, flow, and performance anxiety. Applied Psychology, 62(2), 236-259. https://doi. org/10.1111/j.1464-0597.2012.00494.x

Grant, B. M. (2006). Writing in the company of other women: Exceeding the boundaries. Studies in Higher Education, 31(4), 483-495. https://doi. org/10.1080/03075070600800624

Janke, K. K., Von Hoff, B. et Dy-Boarman, E. (2018). A journey to understand enjoyment in academic writing. INNOVATIONS in pharmacy, 9(3), art. 9. https://doi. org/10.24926/iip.v9i3.1526

Kawabata, M. et Mallett, C. J. (2011). Flow experience in physical activity: Examination of the internal structure of flow from a process-related perspective. Motivation and Emotion, 35(4), 393-402. https://doi.org/10.1007/s11031-011-9221-1

Kempenaar, L. E. et Murray, R. (2016). Writing by academics: a transactional and systems approach to academic writing behaviours. Higher Education Research \& Development, 35(5), 940-950. https://doi.org/10.1080/07294360.2016.1139553

Kornhaber, R., Cross, M., Betihavas, V. et Bridgman, H. (2016). The benefits and challenges of academic writing retreats: An integrative review. Higher Education Research \& Development, 35(6), 1210-1227. https://doi.org/10.1080/07294360.2 $\underline{016.1144572}$

Larivière, V. (2012). On the shoulders of students? The contribution of $\mathrm{PhD}$ students to the advancement of knowledge. Scientometrics, 90(2), 463-481. https://doi. org/10.1007/s11192-011-0495-6

Lee, A. et Boud, D. (2003). Writing groups, change and academic identity: Research development as local practice. Studies in Higher Education, 28(2), 187-200. https://doi.org/10.1080/0307507032000058109 
Levecque, K., Anseel, F., De Beuckelaer, A., Van der Heyden, J. et Gisle, L. (2017). Work organization and mental health problems in $\mathrm{PhD}$ students. Research Policy, 46(4), 868-879. https://doi.org/10.1016/j.respol.2017.02.008

Litalien, D. et Guay, F. (2015). Dropout intentions in PhD studies: A comprehensive model based on interpersonal relationships and motivational resources. Contemporary Educational Psychology, 41, 218-231. https://doi.org/10.1016/j. cedpsych.2015.03.004

Maheux-Pelletier, G., Marsh, H. et Frake-Mistak, M. (2019). The benefits of writing retreats revisited. Dans N. Simmons et A. Singh (dir.), Critical collaborative communities (p. 92-105). Brill Sense. https://doi. org/10.1163/9789004410985 008

Mason, S., Merga, M. K. et Morris, J. E. (2020). Typical scope of time commitment and research outputs of Thesis by Publication in Australia. Higher Education Research \& Development, 39(2), 244-258. https://doi.org/10.1080/07294360.2019.1674253

Mathieu-Chartier, S., Tremblay-Wragg, É., Gadbois, M.-È et Labonté-Lemoyne, É. (2021). Rédiger plus, mieux, ensemble : deux méthodes structurantes pour soutenir la rédaction académique. Dans M.-H. Forget et A. Malo (dir.), (Se) Former à et par l'écriture du qualitatif (p. 1-15). Presses de l'Université Laval.

Mayrath, M. C. (2008). Attributions of productive authors in educational psychology journals. Educational Psychology Review, 20(1), 41-56. https://doi.org/10.1007/ s10648-007-9059-y

Mountz, A., Bonds, A., Mansfield, B. Loyd, J., Hyndman, J., Walton-Roberts, M., Basu, R. Whitson, R., Hawkins, R., Hamilton, T. et Curran, W. (2015). For slow scholarship: A feminist politics of resistance through collective action in the neoliberal university. ACME: An International E-Journal for Critical Geographies, 14(4), 1235-1259. https://www.acme-journal.org/index.php/acme/ article/view/1058

Muthén, L. K. et Muthén, B. O. (1998-2017). Mplus user’s guide (8e éd.). Muthén et Muthén. http://www.statmodel.com/download/usersguide/MplusUserGuideVer_8. pdf 
Papen, U. et Thériault, V. (2018). Writing retreats as a milestone in the development of $\mathrm{PhD}$ students' sense of self as academic writers. Studies in Continuing Education, 40(2), 166-180. https://doi.org/10.1080/0158037X.2017.1396973

Ratković, S., McGinn, M. K., Martinovic, D. et McQuirter Scott, R. (2019). A shared cabin in the Woods: The presence and presents of writing in residential academic writing retreats. Writing \& Pedagogy, 11(2), 165-194. https://doi.org/10.1558/ wap. 35630

Stevenson, N. (2020). Developing academic wellbeing through writing retreats. Journal of Further and Higher Education, 1-13. https://doi.org/10.1080/03098 $\underline{77 X .2020 .1812549}$

Stewart, C. P. (2018). Academic writing retreats for graduate students: a qualitative case study [Thèse de doctorat, Colorado State University]. Mountain Scholar. https://mountainscholar.org/bitstream/handle/10217/189291/Stewart colostate 0053A 14664.pdf? sequence $=1$ \&isAllowed $=\mathrm{y}$

Tremblay-Wragg, É., Mathieu-Chartier, S., Labonté-Lemoyne, É., Déri, C. et Gadbois, M.-È. (2020). Writing more, better, together: How writing retreats support graduate students through their journey. Journal of Further and Higher Education, 45(1), 95-106. https://doi.org/10.1080/0309877X.2020.1736272

Van der Maren, J.-M. (2003). La recherche appliquée en pédagogie : des modèles pour l'enseignement. De Boeck.

Wardale, D., Hendrickson, T., Jefferson, T., Klass, D., Lord, L. et Marinelli, M. (2015). Creating an oasis: Some insights into the practice and theory of a successful academic writing group. Higher Education Research \& Development, 34(6), 1297-1310. https://doi.org/10.1080/07294360.2015.1024621

Zaki, L. (2006). L'écriture d'une thèse en sciences sociales : entre contingences et nécessités. Genèses, 65(4), 112-125. https://doi.org/10.3917/gen.065.0112 